\title{
5-Azacytidine inhibits the proliferation of bladder cancer cells via reversal of the aberrant hypermethylation of the hepaCAM gene
}

\author{
XIAORONG WANG ${ }^{1}$, E. CHEN $^{1}$, XUE YANG $^{1}$, YIN WANG $^{1}$, ZHEN QUAN $^{2}$, \\ XIAOHOU $\mathrm{WU}^{2}$ and CHUNLI LUO ${ }^{1}$
${ }^{1}$ Key Laboratory of Diagnostic Medicine designated by the Chinese Ministry of Education, Chongqing Medical University; ${ }^{2}$ Department of Urology, The First Affiliated Hospital of Chongqing Medical University, Chongqing, P.R. China

Received June 14, 2015; Accepted July 24, 2015

DOI: $10.3892 /$ or.2015.4492

\begin{abstract}
Hepatocyte cell adhesion molecule (hepaCAM), a tumor-suppressor gene, is rarely expressed in bladder carcinoma. However, little is known concerning the mechanisms of low hepaCAM expression in bladder cancer. Abnormal hypermethylation in the promoter plays a crucial role in cancer by silencing tumor-suppressor genes, which is catalyzed by DNA methyltransferases (DNMTs). In the present study, a total of 31 bladder cancer and 22 adjacent tissues were assessed by immunohistochemistry to detect DNMT3A/3B and hepaCAM expression. Methylation of hepaCAM was determined by methylation-specific polymerase chain reaction (MSP). The mRNA and protein levels of DNMT3A/3B and hepaCAM were determined by RT-PCR and western blot analysis after treatment with 5-azacytidine (AZAC). Following AZAC treatment, the proliferation of bladder cancer cells was detected by CCK-8 and colony formation assays. Cell cycle distribution was examined by flow cytometry. To further evaluate the tumor-suppressive roles of AZAC and the involved mechanisms, the anti-tumorigenicity of AZAC was tested in vivo. The expression of DNMT3A/3B protein was markedly increased in the bladder carcinoma tissues $(\mathrm{P}<0.05)$, and had a negative linear correlation with hepaCAM expression in the same patients according to Pearson's analysis ( $\mathrm{r}=-0.7176$ / $-0.7127, \mathrm{P}<0.05$ ). The MSP results indicated that the hepaCAM gene was hypermethylated in three bladder cancer cell lines. Furthermore, we found that downregulation of DNMT3A/3B expression, after treatment with AZAC, reversed the hypermethylation and expression of hepaCAM in bladder cancer
\end{abstract}

Correspondence to: Professor Chunli Luo, Department of Laboratory Diagnosis, Chongqing Medical University, 1 Yixueyuan Road, Yu Zhong, Chongqing 400016, P.R. China

E-mail: luochunli79@126.com

Abbreviations: hepaCAM, hepatocyte cell adhesion molecule; AZAC, 5-azacytidine; DNMT3A/3B, DNA methyltransferase 3A/3B

Key words: DNMT3A/3B, cell proliferation, 5-azacytidine, bladder cancer, hepaCAM cells. In addition, AZAC inhibited the proliferation of bladder cancer cells and arrested cells at the G0/G1 phase. The in vivo results showed that expression of DNMT3A/3B and hepaCAM as well as tumor growth of nude mice were markedly altered which corresponded with the in vitro results. Due to the ability to reactivate expression of hepaCAM and inhibit growth of bladder cancer cells, AZAC may represent an effective treatment for bladder cancer.

\section{Introduction}

Hepatocyte cell adhesion molecule (hepaCAM), also known as GliaCAM, is located on chromosome 11q24 and encodes a putative Ig-like cell adhesion molecule with 416 amino acids. It is a newly identified cell adhesion molecule which belongs to the immunoglobulin superfamily $(1,2)$. It was discovered in normal liver tissues, but is decreased during the development of human hepatocellular carcinoma (3). The loss of hepaCAM in cancer could help to promote tumorigenesis. Overexpression of hepaCAM in cancer cells was found to inhibit cell growth and induce cellular senescence and differentiation $(4,5)$. Our previous studies showed that exogenous hepaCAM inhibits renal cell growth by arresting cells at the G0/G1 phase and promotes c-myc degradation $(6,7)$. We also discovered that overexpression of hepaCAM inhibited the cell proliferation of human bladder carcinoma (8). These findings suggest that hepaCAM acts as a tumor-suppressor gene. Nevertheless, little is known about the mechanisms of low hepaCAM expression in bladder cancer.

Abnormal hypermethylation in the promoter plays a crucial role in cancer by silencing tumor-suppressor genes. Particularly, downregulation of many tumor-suppressor genes and DNA repair genes, such as p16, p15, Rb, VHL, E-cadherin, GSTP1, MGMT and hMLH1, is associated with aberrant methylation in cancer cell lines and primary tumors (9). In mammalians, DNA methylation refers to the addition of a methyl group to the cytosine ring of those cytosines that precede a guanosine (referred to as $\mathrm{CpG}$ dinucleotides) to form 5-methylcytosine (10), which is catalyzed by three different enzymes, DNMT1, DMNT3A and DNMT3B. DNMT3A and DNMT3B are most likely responsible for de novo cytosine methylation at previously unmethylated $\mathrm{CpG}$ sites, whereas the maintenance methyltransferase DNMT1 copies pre-existing 
methylation patterns onto the new DNA strand during DNA replication $(11,12)$.

Abundant evidence shows that DNA methyltransferases (DNMTs) are overexpressed in various types of cancers (13-15). DNMTs have become valuable therapeutical targets of cancers by the use of DNMT inhibitors (DNMTi) (16). 5-Azacytidine (AZAC) and 5-aza-2-deoxycytidine (decitabine), characterized as DNMT inhibitors, were initially used as antimetabolites and cytotoxic agents in phase I/II studies of malignancies $(17,18)$. Meanwhile, aberrant hypermethylation of DNA can be reverted by DNMTi. We hypothesized that overexpression of DNMT3A/3B may also contribute to hepaCAM silencing in bladder cancer.

In the present study, we detected the expression of DNMT3A/3B and hepaCAM in bladder cancer tissues. There was a negative linear correlation between DNMT3A/3B and hepaCAM expression in the same bladder carcinoma patients. High expression of DNMT3A/3B and aberrant hypermethylation in promoter $\mathrm{CpG}$ islands of the hepaCAM gene were found in bladder cancer cell lines. Furthermore, we found that downregulation of DNMT3A/3B expression, after treatment with AZAC, reversed the hypermethylation and expression of hepaCAM. Furthermore, AZAC inhibited the growth of bladder cancer in vitro and in vivo, providing a new insight into the therapeutic strategy of bladder cancer.

\section{Materials and methods}

Tissue specimens. Thirty-one urothelial carcinoma samples and 22 corresponding adjacent tissues were collected from patients who underwent total cystectomy. Nine patients were treated with transurethral resection of bladder carcinoma but no adjacent tissue was obtained. Patients were enrolled at the Department of Urology at the First Affiliated Hospital of Chongqing Medical University. All tissue specimens were confirmed to be bladder cancer or normal histologically, and histological grade and stage were determined according to UICC guidelines. Written informed consent was received from all participants. This study was approved by the Ethics Committee of Chongqing Medical University. All tissue specimens were stored at $-80^{\circ} \mathrm{C}$ before the experiment.

Immunohistochemistry. The assay was performed as described previously (19). In brief, paraffin wax-embedded tissue sections were dewaxed, rehydrated and microwaved for $30 \mathrm{~min}$ in sodium citrate buffer to retrieve antigen epitopes. Endogenous peroxidase activity was suppressed by $3 \% \mathrm{H}_{2} \mathrm{O}_{2}$ and blocked by goat serum $5 \%$ BSA. Diluted primary polyclonal rabbit antibodies against hepaCAM (ProteinTech, China), DNMT3A and DNMT3B (Immunoway Biotechnology, China) were added and left at $4^{\circ} \mathrm{C}$ overnight. As secondary reagents, we used biotin-labeled anti-IgG and avidin-biotin horseradish peroxidase complex, followed by staining with the chromogen diaminobenzidine (Zhongshan, China) until a brown color developed. Slides were counterstained with Mayer's hematoxylin and differentiated in a solution containing $1 \%$ hydrochloric acid and $99 \%$ ethanol. Cell nuclei were stained blue using lithium carbonate. Sections were dehydrated and a transparent coverslip was added to enable observation by microscopy.
Cells and culture. Human bladder cancer cell lines (T24, EJ and BIU-87) were obtained from the American Type Culture Collection (ATCC; USA). All the cells were cultured in RPMI-1640 medium supplemented with $10 \%$ fetal bovine serum (both from Gibco, USA) under standard culture conditions $\left(5 \% \mathrm{CO}_{2}\right.$ at $\left.37^{\circ} \mathrm{C}\right)$.

MTT assay. To determine the optimal drug doses, bladder cancer cells (T24, EJ and BIU-87) were seeded in 96-well plates at a density of $1 \times 10^{4}$ cells/well for $12 \mathrm{~h}$ and treated with 5 -azacytidine (Sigma, USA) at different concentrations $(0.5$, 1, 2, 3, 4, 5, 6 and $7 \mu \mathrm{g} / \mathrm{ml})$. Three plates were seeded, and the cells were cultured for 24, 48, 72 and $96 \mathrm{~h}$, respectively. Before removal from the incubator, the cells were incubated with $20 \mu 10.5 \mathrm{mg} / \mathrm{ml}$ MTT (Sigma) for an additional $4 \mathrm{~h}$. Culture medium was discarded, and $150 \mu 1$ dimethylsulfoxide (DMSO; Sigma) was added to dissolve the formazan crystals. The absorbance was measured in a microplate reader at an optical density (OD) of $492 \mathrm{~nm}$. Inhibition rate was calculated using the formula: IR = $(1$ - experiment group/control group $)$ $\mathrm{x} 100 \%$.

$C C K-8$ assay. To evaluate the cell viability, the CCK-8 Kit (Beyotime, China) was applied according to the manufacturer's instructions. The results were repeated in triplicate. The OD of the untreated controls was measured as $100 \%$ survival.

Colony formation assay. Bladder cancer cells were seeded into 6-well plates at a density of 300 cells/well. After a 48-h incubation, cells were treated with AZAC or DMSO. After 13 days, the cells were fixed with methanol for $20 \mathrm{~min}$ and stained with crystal violet. Colonies were observed and images were captured under a microscope.

Flow cytometric assay. Bladder cancer cells $\left(1 \times 10^{6}\right)$ treated with AZAC or DMSO were cultured in 6-well plates for $48 \mathrm{~h}$. The cells were collected in cold phosphate-buffered solution (PBS), fixed in $70 \%$ ethanol, and stored at $4^{\circ} \mathrm{C}$ for subsequent cell cycle analysis. Procedures for testing the cell cycle have been previously described (7).

Methylation-specific polymerase chain reaction assay. Genomic DNA was extracted from bladder cancer cells according to the instructions provided in the TIANamp Genomic DNA kit (Tiangen Biotech, China). The bisulfite modification procedures were carried out using EZ DNA Methylation-Gold ${ }^{\mathrm{TM}}$ kit (Zymo Research, USA). Methylationspecific polymerase chain reaction (MSP) was performed to detect the methylation status of the hepaCAM gene (M, methylation status; $\mathrm{U}$, unmethylation status). The PCR conditions were as follows: pre-denaturation at $98^{\circ} \mathrm{C}$ for $10 \mathrm{~min}$, denaturation at $95^{\circ} \mathrm{C}$ for $10 \mathrm{sec}$; annealing at $58.4^{\circ} \mathrm{C}(\mathrm{U}) / 61.8^{\circ} \mathrm{C}(\mathrm{M})$ for $60 \mathrm{sec}$; extension at $72^{\circ} \mathrm{C}$ for $30 \mathrm{sec}$, final extension at $72^{\circ} \mathrm{C}$ for $5 \mathrm{~min}$. Primer sequences were: hepaCAM(M) forward, AGAA TTCGGTTTCGGAGTTTC and reverse, CTAAACGACGAC GAATATATCCG; and hepaCAM(U) forward, AGAATTTGG TTTTGGAGTTTTGA and reverse, CCTAAACAACAACAA ATATATCCAAC. PCR products were separated on $3 \%$ agarose gel. 
A

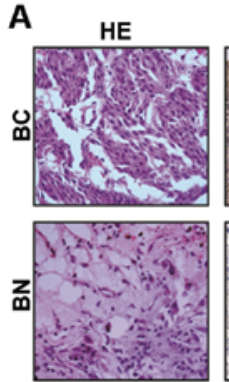

C
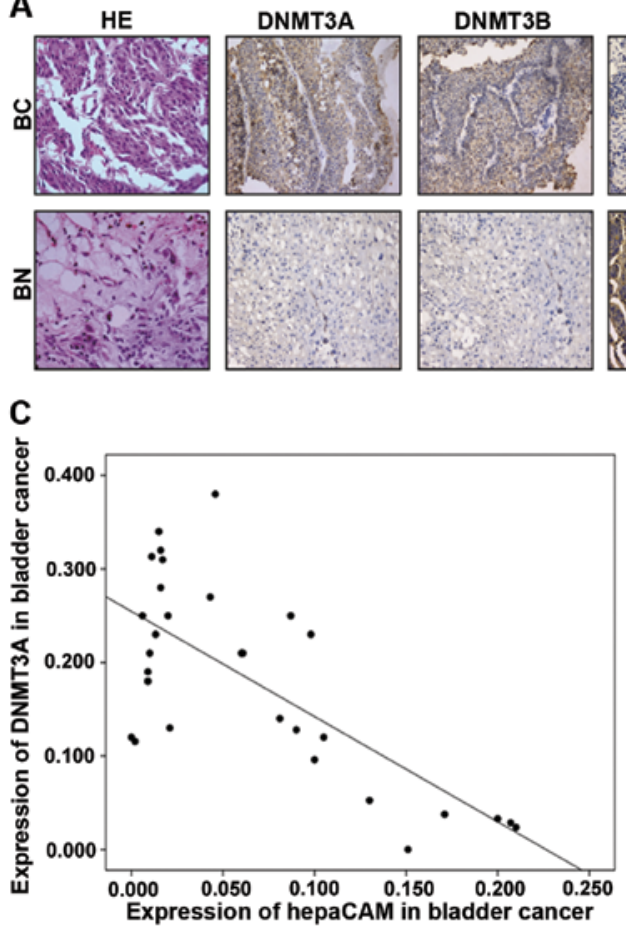

D

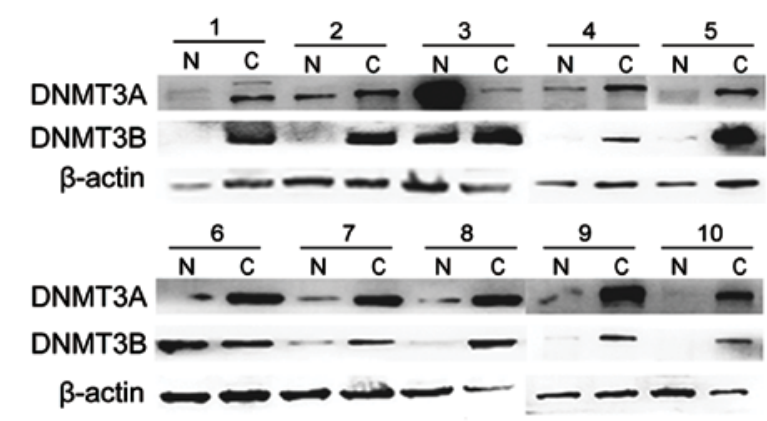

\section{E}
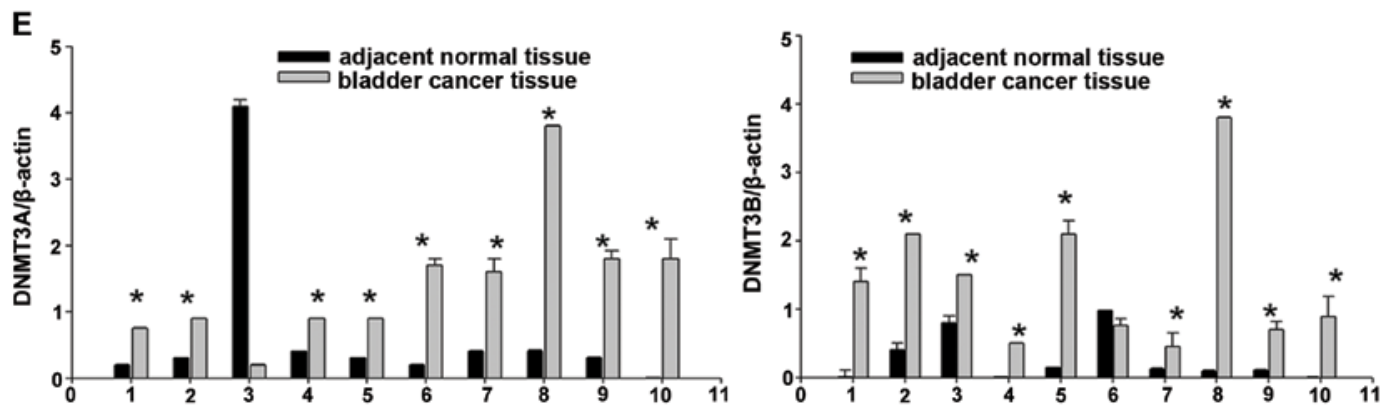

Figure 1. Expression levels of DNMT3A/3B and hepaCAM in bladder cancer and adjacent non-malignant tissues were determined using immunohistochemistry. (A) High DNMT3A/3B expression but low hepaCAM expression was noted in the bladder cancer tissues. Tissue samples from 31 patients diagnosed with bladder carcinoma were assessed by immunohistochemical (IHC) staining with DNMT3A, DNMT3B and hepaCAM antibodies. Original magnification, x200. (B) The relative expression of DNMT3A/3B and hepaCAM in bladder cancer tissues. (C) The correlation curve shows hepaCAM vs. DNMT3A/3B expression in bladder cancer specimens using Pearson's analysis ( $\mathrm{r}=-0.7176 /-0.7127, \mathrm{P}<0.05)$. (D and E) Protein lysates extracted from 10 bladder cancer tissue samples were immunoblotted with antibodies against DNMT3A/3B (N, adjacent normal tissues; C, bladder cancer tissues). Quantification of protein from three separate experiments and normalized to $\beta$-actin is shown. Data represent means \pm SDs of three independent experiments. ${ }^{*}<0.05$, compared with the adjacent normal tissues.

Reverse transcription and RT-PCR. Total RNA was isolated from bladder cancer cells using RNAiso Plus (Takara, Japan) according to the manufacturer's instructions. Complementary DNA was synthesized using a reverse transcription kit (Takara) according to the manufacturer's protocol. The PCR conditions consisted of predenaturing at $95^{\circ} \mathrm{C}$ for $5 \mathrm{~min}$, denaturing at $95^{\circ} \mathrm{C}$ for $10 \mathrm{sec}$, annealing for $50 \mathrm{sec}$, extension at $72^{\circ} \mathrm{C}$ for $1 \mathrm{~min}$, a total of 35 cycles from denaturing, and a final extension at $72^{\circ} \mathrm{C}$ for $5 \mathrm{~min}$. Primer sequences were as follows: hepaCAM forward, TACTGTAGATGTGCCCATTTCG and 
Table I. Comparison of the protein expression of DNMT3A and hepaCAM in the bladder cancer cases according to clinicopathological parameters.

\begin{tabular}{|c|c|c|c|c|c|c|c|c|c|}
\hline \multirow[b]{2}{*}{ Variable } & \multirow[b]{2}{*}{$\mathrm{n}(\%)$} & \multicolumn{2}{|c|}{ DNMT3A } & \multicolumn{2}{|c|}{ hepaCAM } & \multicolumn{2}{|c|}{ Mean density (mean \pm SD) } & \multicolumn{2}{|c|}{ P-value } \\
\hline & & - & + & - & + & DNMT3A & hepaCAM & DNMT3A & hepaCAM \\
\hline Tissue & & & & & & & & $0.008^{\mathrm{a}}$ & $0.006^{\mathrm{a}}$ \\
\hline Cancer & 31 & 6 & 25 & 27 & 4 & $0.182 \pm 0.104$ & $0.065 \pm 0.066$ & & \\
\hline Adjacent & 22 & 20 & 2 & 3 & 19 & $0.076 \pm 0.071$ & $0.193 \pm 0.119$ & & \\
\hline Gender & & & & & & & & 0.067 & 0.116 \\
\hline Male & $26(84)$ & 5 & 21 & 24 & 2 & $0.183 \pm 0.112$ & $0.066 \pm 0.045$ & & \\
\hline Female & $5(16)$ & 1 & 4 & 3 & 2 & $0.181 \pm 0.143$ & $0.064 \pm 0.093$ & & \\
\hline Age (years) & & & & & & & & 0.079 & 0.117 \\
\hline$\geq 60$ & $21(68)$ & 5 & 16 & 17 & 4 & $0.183 \pm 0.161$ & $0.065 \pm 0.091$ & & \\
\hline$<60$ & $10(32)$ & 1 & 9 & 10 & 0 & $0.182 \pm 0.132$ & $0.064 \pm 0.063$ & & \\
\hline Histological stage & & & & & & & & 0.346 & 0.211 \\
\hline Ta-T1 & $9(29)$ & 5 & 4 & 8 & 1 & $0.181 \pm 0.152$ & $0.064 \pm 0.054$ & & \\
\hline $\mathrm{T} 2-\mathrm{T} 4$ & $22(71)$ & 1 & 21 & 19 & 3 & $0.182 \pm 0.141$ & $0.065 \pm 0.028$ & & \\
\hline Histological grade & & & & & & & & 0.098 & 0.113 \\
\hline G1-G2 & $21(68)$ & 5 & 16 & 21 & 0 & $0.182 \pm 0.133$ & $0.064 \pm 0.019$ & & \\
\hline G3-G4 & $10(32)$ & 1 & 9 & 6 & 4 & $0.183 \pm 0.167$ & $0.066 \pm 0.021$ & & \\
\hline Occurrence & & & & & & & & 0.198 & 0.214 \\
\hline Primary & $20(65)$ & 5 & 15 & 19 & 1 & $0.182 \pm 0.107$ & $0.064 \pm 0.091$ & & \\
\hline Recurrent & $11(35)$ & 1 & 10 & 8 & 3 & $0.184 \pm 0.171$ & $0.065 \pm 0.089$ & & \\
\hline
\end{tabular}

${ }^{\mathrm{a}} \mathrm{P}<0.05$, considered significant.

reverse, CTTCTGGTTTCAGGCGGTC; DNMT3A forward, GGCGTTAGTGACAAGAGGG and reverse, TGGACTGGG AAACCAAATA; DNMT3B forward, CAAAGAGTTGGGC ATAAAGG and reverse, GCGTGAGTAATTCAGCAGGT; and $\beta$-actin forward, CACCACACCTTCTACAATGAGC and reverse, GTGATCTCCTTCTGCATCCTGT. The $\beta$-actin gene was used as an internal standard. All RT-PCR reactions were executed in triplicate. Each PCR product was electrophoretically tested on $1.5 \%$ agarose gel.

Protein extraction and western blot analysis. Total protein extraction from cells and tissues was performed using RIPA buffer supplemented with protease inhibitor (PMSF) and phosphatase inhibitors $\left(\mathrm{NaF}\right.$ and $\mathrm{Na}_{3} \mathrm{VO}_{4}$ ) (Roche, Switzerland). The BCA Protein Assay kit (Beyotime) was used to detect the quantity of each sample according to the manufacturer's instructions. Proteins $(100 \mu \mathrm{g})$ were separated by $10 \%$ sodium dodecyl sulfate-polyacrylamide gel electrophoresis (Invitrogen, USA). Then the proteins were electrotransferred onto polyvinylidene difluoride membranes (Millipore, USA). The membranes were blocked with 5\% skim milk at room temperature for $1 \mathrm{~h}$ and then incubated with the primary antibody against hepaCAM, DNMT3A, DNMT3B and $\beta$-actin (Zhongshan, China) overnight at $4^{\circ} \mathrm{C}$. Membranes were washed twice with TBST and once with TBS, and then incubated with the HRP-conjugated secondary antibody for $1 \mathrm{~h}$ at room temperature. Proteins were visualized using a chemiluminescence (ECL) reagent (Millipore, Billerica, MA, USA), and the densities of the bands were quantified and normalized to that of $\beta$-actin by Quantity One software. The experiments were performed as described previously (20).

Tumor model. Nude mice (4-6 weeks of age) were purchased from the Animal Institute of the Chinese Medical Academy (Beijing, China). Viable EJ cells $\left(3 \times 10^{6}\right)$ resuspended in PBS were injected into the right flanks of 8 male nude mice. The mice were randomized into two groups: group A (DMSO group) and group B (AZAC group) after 2 weeks. Subsequently, AZAC was injected into mice in group B and DMSO in group A every three days for 6 times. The weight of each nude mice was determined daily for 3 weeks. After 3 weeks, all mice were sacrificed. The xenograft tumor weight was measured at the terminal time, and tumor tissues were resected for hematoxylin and eosin (H\&E) staining and immunohistochemical examination of hepaCAM, DNMT3A and DNMT3B. All animal experiments were approved by the Institutional Animal Care and Use Committee (IACUC) of Chongqing Medical University.

Statistical analysis. All statistical analyses were performed with SPSS 19.0 software using paired-samples t-test. Data are shown as mean $\pm \mathrm{SD}$, and $\mathrm{P}<0.05$ served as the criterion for statistical significance. 
Table II. Comparison of the protein expression of DNMT3B and hepaCAM in the bladder cancer cases according to clinicopathological parameters.

\begin{tabular}{|c|c|c|c|c|c|c|c|c|c|}
\hline \multirow[b]{2}{*}{ Variable } & \multirow[b]{2}{*}{$\mathrm{n}(\%)$} & \multicolumn{2}{|c|}{ DNMT3B } & \multicolumn{2}{|c|}{ hepaCAM } & \multicolumn{2}{|c|}{ Mean density (mean \pm SD) } & \multicolumn{2}{|c|}{ P-value } \\
\hline & & - & + & - & + & DNMT3B & hepaCAM & DNMT3B & hepaCAM \\
\hline Tissue & & & & & & & & $0.011^{\mathrm{a}}$ & $0.006^{\mathrm{a}}$ \\
\hline Cancer & 31 & 5 & 26 & 27 & 4 & $0.176 \pm 0.094$ & $0.065 \pm 0.066$ & & \\
\hline Adjacent & 22 & 20 & 2 & 3 & 19 & $0.073 \pm 0.061$ & $0.193 \pm 0.119$ & & \\
\hline Gender & & & & & & & & 0.087 & 0.116 \\
\hline Male & $26(84)$ & 4 & 22 & 24 & 2 & $0.175 \pm 0.131$ & $0.066 \pm 0.045$ & & \\
\hline Female & $5(16)$ & 1 & 4 & 3 & 2 & $0.177 \pm 0.123$ & $0.064 \pm 0.093$ & & \\
\hline Age (years) & & & & & & & & 0.093 & 0.117 \\
\hline$\geq 60$ & $21(68)$ & 4 & 17 & 17 & 4 & $0.178 \pm 0.114$ & $0.065 \pm 0.091$ & & \\
\hline$<60$ & $10(32)$ & 1 & 9 & 10 & 0 & $0.175 \pm 0.123$ & $0.064 \pm 0.063$ & & \\
\hline Histological stage & & & & & & & & 0.199 & 0.211 \\
\hline Ta-T1 & $9(29)$ & 4 & 5 & 8 & 1 & $0.175 \pm 0.127$ & $0.064 \pm 0.054$ & & \\
\hline $\mathrm{T} 2-\mathrm{T} 4$ & $22(71)$ & 1 & 21 & 19 & 3 & $0.177 \pm 0.161$ & $0.065 \pm 0.028$ & & \\
\hline Histological grade & & & & & & & & 0.099 & 0.113 \\
\hline G1-G2 & $21(68)$ & 3 & 18 & 21 & 0 & $0.175 \pm 0.132$ & $0.064 \pm 0.019$ & & \\
\hline G3-G4 & $10(32)$ & 2 & 8 & 6 & 4 & $0.178 \pm 0.176$ & $0.066 \pm 0.021$ & & \\
\hline Occurrence & & & & & & & & 0.215 & 0.214 \\
\hline Primary & $20(65)$ & 4 & 16 & 19 & 1 & $0.175 \pm 0.161$ & $0.064 \pm 0.091$ & & \\
\hline Recurrent & $11(35)$ & 1 & 10 & 8 & 3 & $0.177 \pm 0.175$ & $0.065 \pm 0.089$ & & \\
\hline
\end{tabular}

${ }^{\mathrm{a}} \mathrm{P}<0.05$, considered significant.

\section{Results}

High expression of DNMT3A/3B and low expression of hepaCAM are noted in the bladder cancer tissues. To investigate whether there was any correlation between DNMT3A/3B and hepaCAM expression, we used anti-DNMT3A/3B and anti-hepaCAM antibodies to detect the expression of DNMT3A/3B and hepaCAM in the bladder cancer and adjacent tissues. The results showed that DNMT3A/3B was strongly expressed in the bladder cancer tissues, but weakly expressed in the adjacent tissues. However, in regards to hepaCAM, the expression levels in the adjacent tissues were higher than levels in the cancer tissues (Fig. 1A and B). We made use of the mean density for estimating the protein expression levels of DNMT3A/3B and hepaCAM. The results revealed a negative linear correlation between DNMT3A/3B and hepaCAM expression in the same patients according to Pearson's analysis ( $r=-0.7176 /-0.7127, \mathrm{P}<0.05$, Fig. 1C). However, there was no significant difference in expression levels in regards to age, gender, and disease recurrence (Tables I and II).

In contrast, protein lysates were extracted from 10 bladder cancer patient samples. Importantly, western blotting with antibodies against DNMT3A/3B showed that 9 of the 10 bladder cancer tissues had elevated levels of DNMT3A/3B, respectively, whereas only 1 of the 10 adjacent normal tissues had detectable expression of DNMT3A/3B $(\mathrm{P}<0.05$, Fig. 1D and E).
Optimal concentration and treatment time for AZAC. To confirm the mode of action of AZAC in bladder cancer cells, we determined the absorption at a variety of concentrations and times. We calculated the IR with the above-documented equation. The results indicated that bladder cancer cell proliferation was markedly inhibited by AZAC in a concentration- and dosedependent manner $(\mathrm{P}<0.05$, Table III). According to Table III, $4 \mu \mathrm{g} / \mathrm{ml}$ (BIU-87) and $5 \mu \mathrm{g} / \mathrm{ml}$ (T24 and EJ) was confirmed as the most appropriate concentrations. The dose-effect curve indicated that the bladder cancer cell IR gradually increased with prolongation of treatment time at the same concentration compared with the controls $(\mathrm{P}<0.05$, Fig. $2 \mathrm{~A})$. Based on these results, concentrations of $4 \mu \mathrm{g} / \mathrm{ml}$ (BIU-87 cells) and $5 \mu \mathrm{g} / \mathrm{ml}$ (T24 and EJ cells) AZAC were selected as the optimal treatment conditions for the following experiments.

$A Z A C$ reverses the hypermethylation of hepaCAM. Hypermethylation of the hepaCAM promoter was apparently observed in the T24, EJ and BIU-87 cell lines by use of MSP. In addition, the hepaCAM promoter was obviously demethylated by $5 \mu \mathrm{g} / \mathrm{ml}$ of AZAC in the T2 4 and EJ cell lines, and $4 \mu \mathrm{g} / \mathrm{ml}$ of AZAC in the BIU-87 cell line at $48 \mathrm{~h}$ (Fig. 2B).

Suppression of DNMT3A/3B induces re-expression of hepaCAM. Based on our findings, we analyzed the mRNA and protein expression of DNMT3A/3B and hepaCAM by RT-PCR and immunoblotting. The results revealed that DNMT 
Table III. Inhibition rates for different concentrations of AZAC in the bladder cancer cells.

AZAC concentration

\begin{tabular}{lccrrrrrr}
\cline { 2 - 8 } Cancer cells & 0.5 & 1 & 2 & 3 & 4 & 5 & 6 & 7 \\
\hline T24 & & & & 25.05 & 27.34 & 32.25 & 39.17 & 44.56 \\
EJ & & & & 3.63 & 6.96 & 33.00 & 34.86 & 35.87 \\
BIU-87 & 8.77 & 12.75 & 12.93 & 28.33 & 34.70 & 48.15 & &
\end{tabular}

Note: concentration, $\mu \mathrm{g} / \mathrm{ml}$; inhibition rate, $\%$.

inhibitor AZAC inhibited both the mRNA and protein levels of DNMT3A/3B in the bladder cancer cell lines. AZAC not only reversed the expression of hepaCAM mRNA (Fig. 2C and D) but also its protein level (Fig. 2E and F). These results suggest that the inactivation of DNMT3A/3B by AZAC treatment may participate in restoring the expression of the tumor-suppressor gene hepaCAM.

AZAC inhibits cell proliferation. To investigate the effect of AZAC against bladder cancer cells, cell proliferation was detected by CCK- 8 assay, colony formation assay and flow cytometry. The results showed that the growth of bladder cancer cells was inhibited after exposure to AZAC for $48 \mathrm{~h}$ compared with the control groups $(\mathrm{P}<0.05$, Fig. 3A). This result was further supported by a colony formation assay. As shown in Fig. 3B and C, there was a significantly lower colony formation potential in the AZAC-treated cells compared to that in the DMSO treatment groups $(\mathrm{P}<0.05)$.

It has been reported that the growth inhibition of AZAC is always associated with cell cycle arrest (21). To evaluate the exact cell cycle phase, bladder cancer cells were inspected at $48 \mathrm{~h}$, under stimulation by $4 \mu \mathrm{g} / \mathrm{ml}$ (BIU-87) and $5 \mu \mathrm{g} / \mathrm{ml}$ (T24 and EJ) AZAC. The results revealed that bladder cancer cells treated with AZAC had a significant accumulation in the G0/G1 phase compared to the controls $(\mathrm{P}<0.05$, Fig. 3D and E). These data indicate that the growth of bladder cancer cells was markedly inhibited by AZAC.

Effects of AZAC on nude mice. To further investigate the tumorsuppressive function of AZAC and its mechanisms in vivo, the antitumorigenicity of AZAC was tested in nude mice. Thirty days after injection, tumors were excised from the tested mice for further analysis. The volume and weight of the tumors in the AZAC treatment groups were significantly decreased, compared with the control groups $(\mathrm{P}<0.05$, Fig. 4A-D). Immunohistochemistry was further performed to analyze the expression of DNMT3A/3B and hepaCAM in the xenograft tumors. Numerous tumor cells with high nuclear fragmentation were found in H\&E-stained sections from the DMSO treatment groups compared with the AZAC groups. Furthermore, low expression of DNMT3A/3B but high levels of hepaCAM immunostaining were observed in the AZAC treatmentderived tumor tissues (Fig. 4E). Taken together, these data indicate that AZAC acts as an antitumor agent, suppressing the proliferation of bladder cancer and enhancing the expression of hepaCAM via downregulation of the levels of DNMT3A/3B.

\section{Discussion}

Bladder cancer ranks fourth in regards to the worldwide cancer incidence and is the seventh leading cause of mortality from cancer (22). The main features of bladder cancer are disease recurrence and progression (23). Research has confirmed that bladder cancer results from interactions among exogenous, genetic and epigenetic factors. Previous studies have demonstrated that the most common risk factors of bladder cancer are smoking and occupational exposure (23-25). However, more and more studies have aimed to investigate the molecular mechenisms of bladder carcinogenesis in recent years. Various studies have discovered that inactivation of one or more tumorsuppressor genes, such as p16INK4a, FHIT, LAMC2, APC, hMLH1 and E-cadherin, may be an early event in the tumorigenic pathway leading to bladder cancer (26-29).

HepaCAM localized on the cell surface has a role in cell-cell adhesion, and acts as a tumor suppressor. We have been researching the reason for the absence of hepaCAM in bladder cancer. In a previous study, we found that exon 2 methylation of hepaCAM may be one of the reasons for its low expression in bladder cancer tissues and cell lines (30). However, the precise mechanism of low hepaCAM expression in bladder cancer still needs to be elucidated.

In cancer, hypermethylation of $\mathrm{CpG}$ islands, regarded as regions of DNA $0.5-4 \mathrm{~kb}$ with $\mathrm{C}+\mathrm{G}$ content $>0.5$ (31-33), contributes to transcription silencing of tumor-suppressor genes. There is mounting evidence that abnormal hypermethylation of the promoter is the mechanism involved in the silencing of tumor-suppressor genes in tumorigenesis. For example, RASSF1A is methylated in lung and breast cancers, and hypermethylation of the $\mathrm{CDH} 1$ promoter is noted in gastric cancer (34). In the past few years, more and more candidate tumor-suppressor genes have been found to be silenced by promoter hypemethylation in cancers. In the present study, we predicted a CpG island in the hepaCAM promoter by an online database (http://rulai.cshl.org/tools/FirstEF/) and designed meth-primers (http://www.urogene.org/methprimer/index1. html) to detect the methylation status of the $\mathrm{CpG}$ island in the hepaCAM promoter. Our results demonstrated that aberrant hypermethylation occurred in the $\mathrm{CpG}$ island of the hepaCAM gene promoter, which contributed to the absence of hepaCAM in the bladder cancer cells (T24, EJ and BIU-87). Methylation was catalyzed by DNMTs. Many previous studies have shown that DNMT3A/3B are overexpressed in cancers. For example, the level of DNMT3A/3B was signifi- 
A

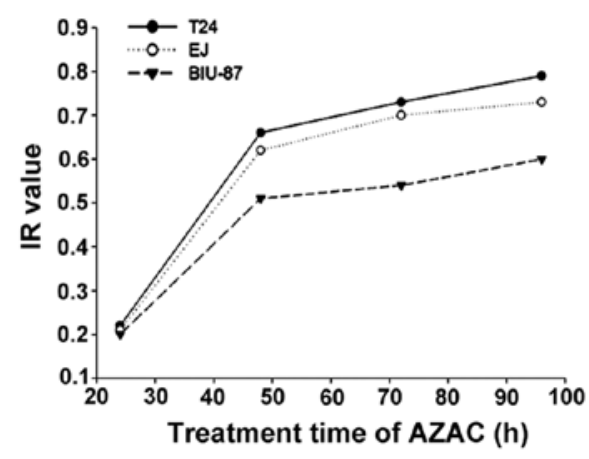

B

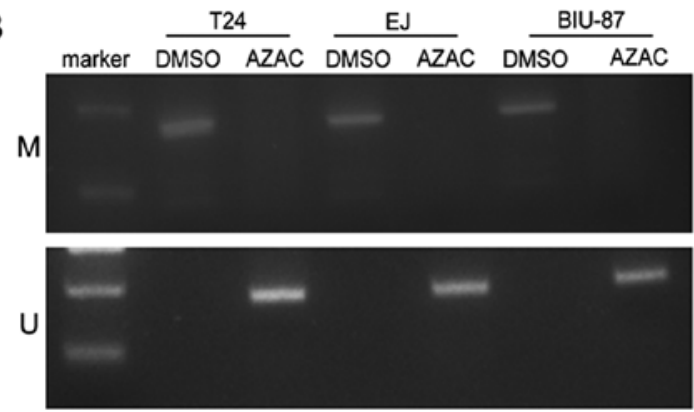

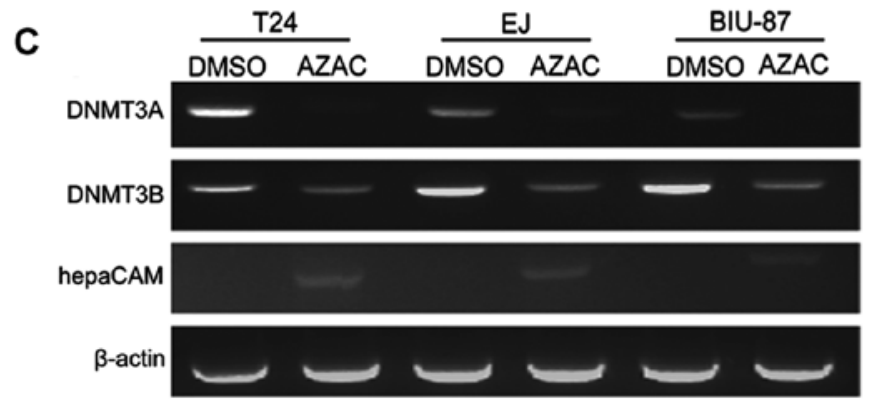

D
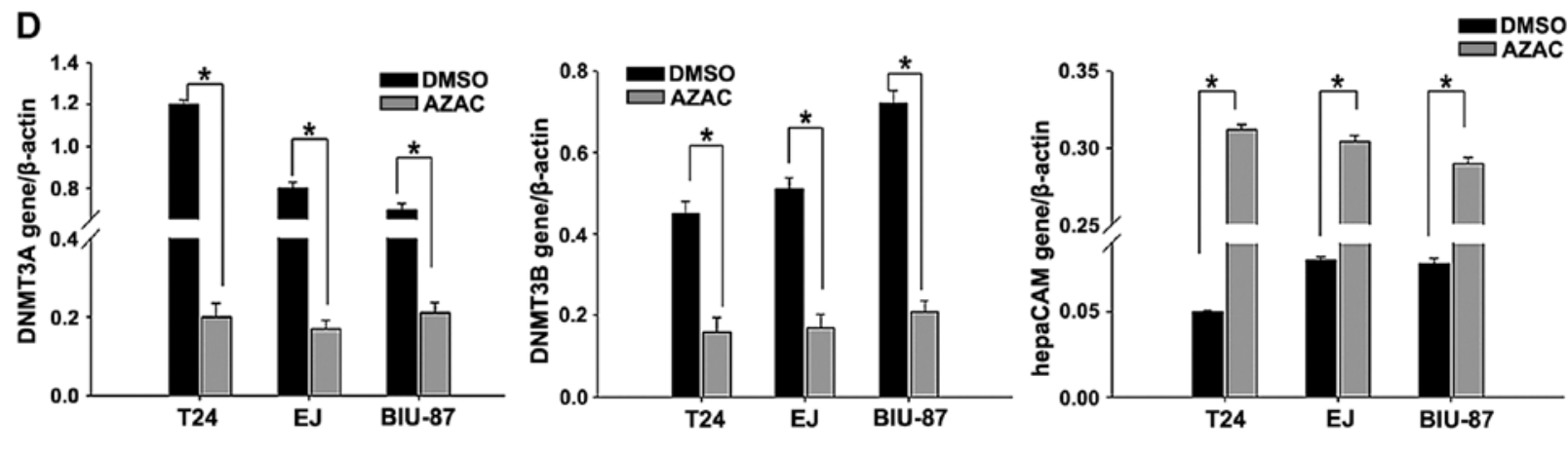

E $\frac{\text { T24 }}{\text { DMSO AZAC DMSO AZAC DMSO AZAC }}$

DNMT3A

$\beta$-actin

DNMT3B

$\beta$-actin hepaCAM $\beta$-actin
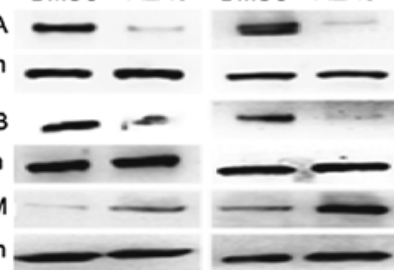

F
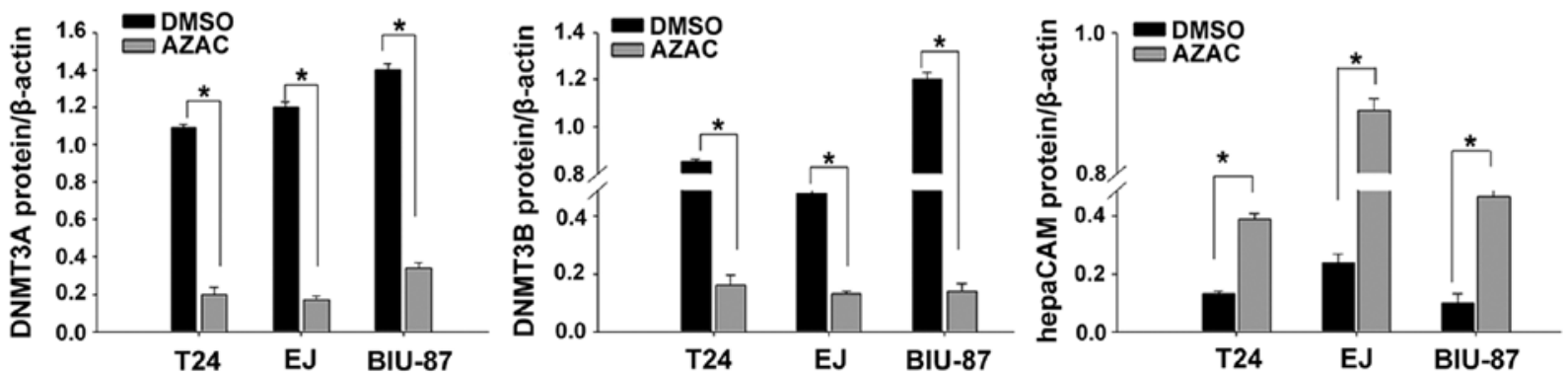

Figure 2. AZAC reverses hepaCAM methylation and AZAC reduces levels of DNMT3A/3B and induces expression of hepaCAM. (A) MTT assay results revealed that the DNMT inhibitor, AZAC, increased the bladder cancer cell inhibition rate in a time-dependent manner. Time-points included $24,48,72$ and $96 \mathrm{~h}$. AZAC at $4 \mu \mathrm{g} / \mathrm{ml}$ (BIU-87) and $5 \mu \mathrm{g} / \mathrm{ml}$ (T24 and EJ) for $48 \mathrm{~h}$ were the optimal concentrations and times for use in the experiment. (B) MSP analysis of the methylation of the hepaCAM gene in bladder cancer cells. We found that in the blank group, hepaCAM was hypermethylated which was reversed after treatment of AZAC compared with the DMSO group (M, methylation; U, unmethylation). (C) RT-PCR was used to detect mRNA expression of DNMT3A, DNMT3B and hepaCAM. HepaCAM expression was upregulated and DNMT3A/3B expression was decreased significantly compared with the DMSO group. (D) Relative intensities of target bands of PCR were scanned by Quantity One software; * $\mathrm{P}<0.05$, compared with the DMSO group. (E) Western blot analysis showed that hepaCAM was re-activated but DNMT3A and DNMT3B were downregulated in three bladder cancer cell lines after AZAC treatment compared with the DMSO group. $\beta$-actin was used as control. (F) Intensities were quantitated by Quantity One software; ${ }^{*} \mathrm{P}<0.05$, compared with the DMSO group. 


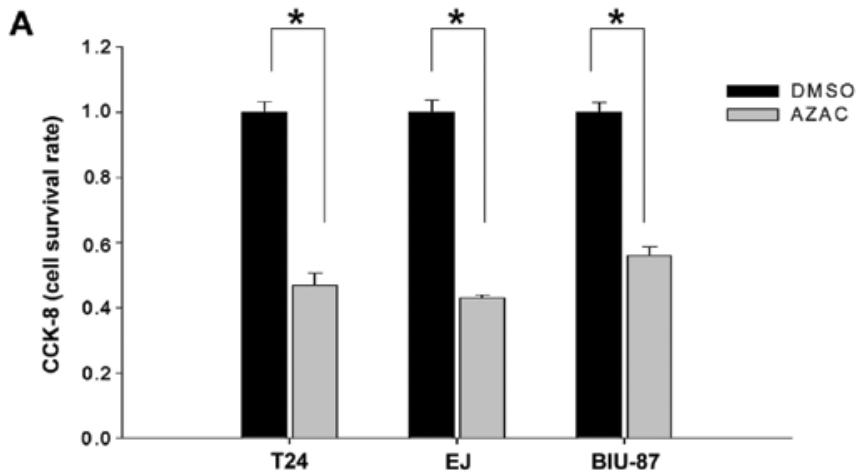

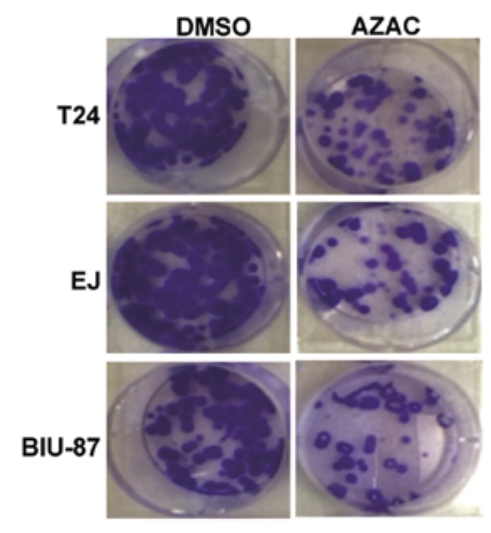

D

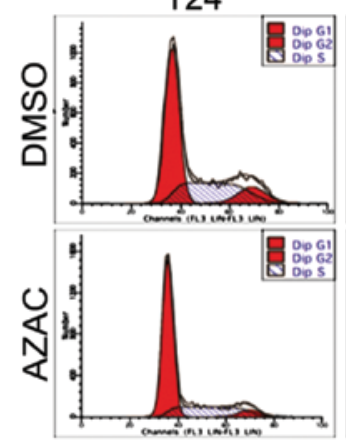

C

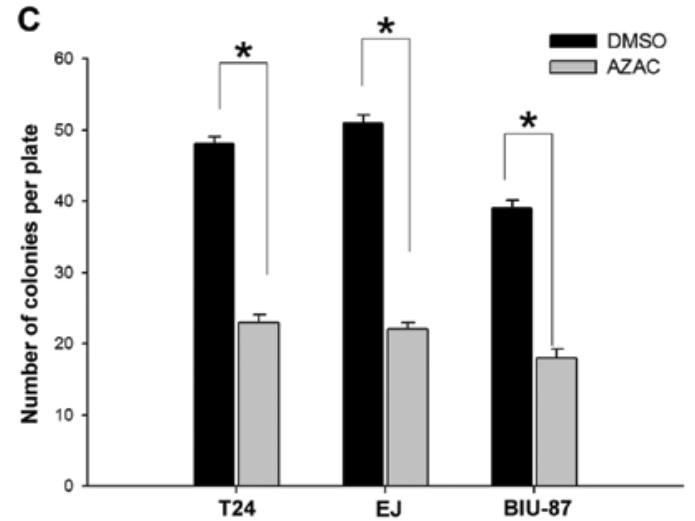

EJ

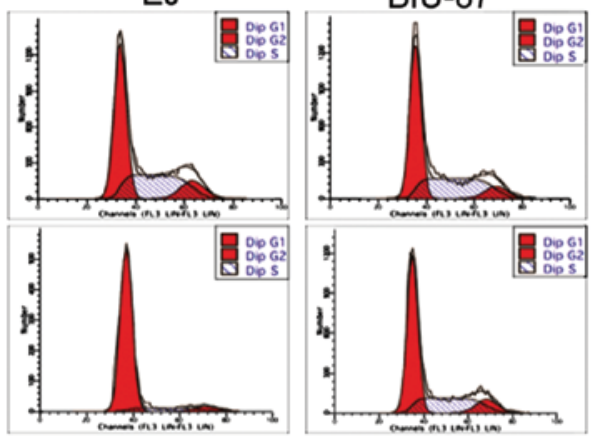

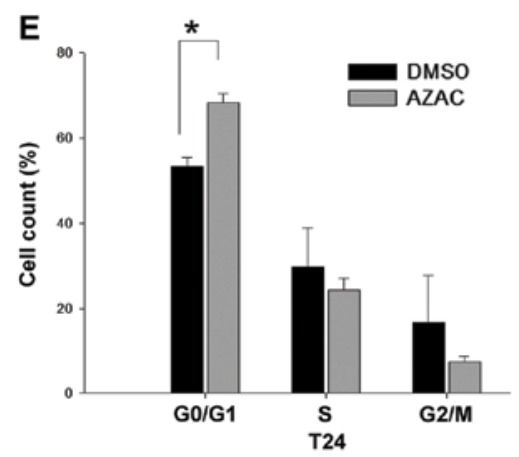
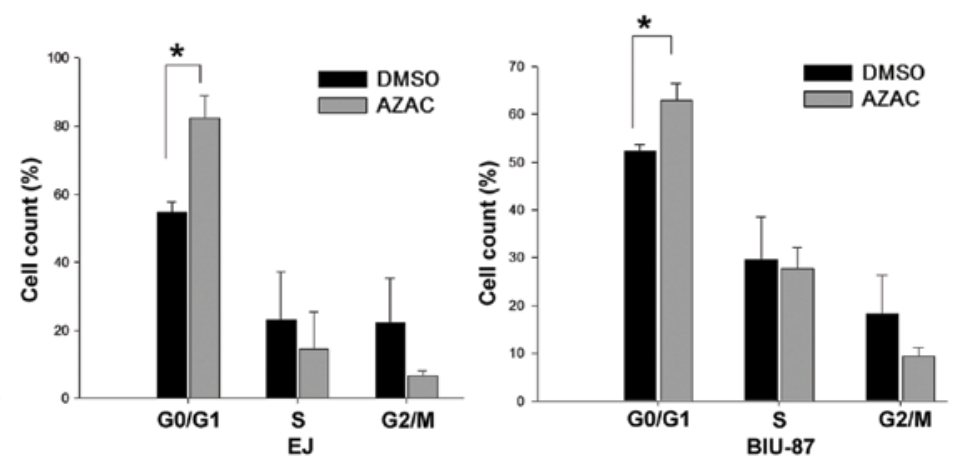

Figure 3. AZAC inhibits the proliferation of bladder cancer cells. (A) CCK-8 assay was performed to investigate the cell viability after treatment with AZAC for $48 \mathrm{~h}$. The results showed that proliferation of the bladder cancer cells was inhibited after AZAC treatment; P<0.05, compared with the control group. (B and C) The number of colonies was decreased substantially after exposure to AZAC when compared with DMSO; ${ }^{*} \mathrm{P}<0.05$. (D) Flow cytometry revealed an increased number of cells in the G0/G1 phase and a decreased number in the S and G2 phase in bladder cancer cells stimulated with AZAC for 48 h. (E) Cell percentage in each phase according to flow cytometry $(\mathrm{FCM})$. Data are reported as mean $\pm \mathrm{SD}(\mathrm{n}=3)$, compared with the control group; ${ }^{*} \mathrm{P}<0.05$.

cantly higher in gastric cancer than in normal tissues (35), and its expression in prostate carcinoma tissues was higher than that in adjacent normal tissues (36). In the present study, we detected the expression of DNMT3A/3B and hepaCAM in bladder cancer. Correlations between DNMT3A/3B and
hepaCAM in bladder cancer patients suggested that a low level of hepaCAM was closely related with high expression of DNMT3A/3B in bladder carcinomas. Then, we found that AZAC reversed the high methylation of hepaCAM and restored its level via downregulation of the expression of 

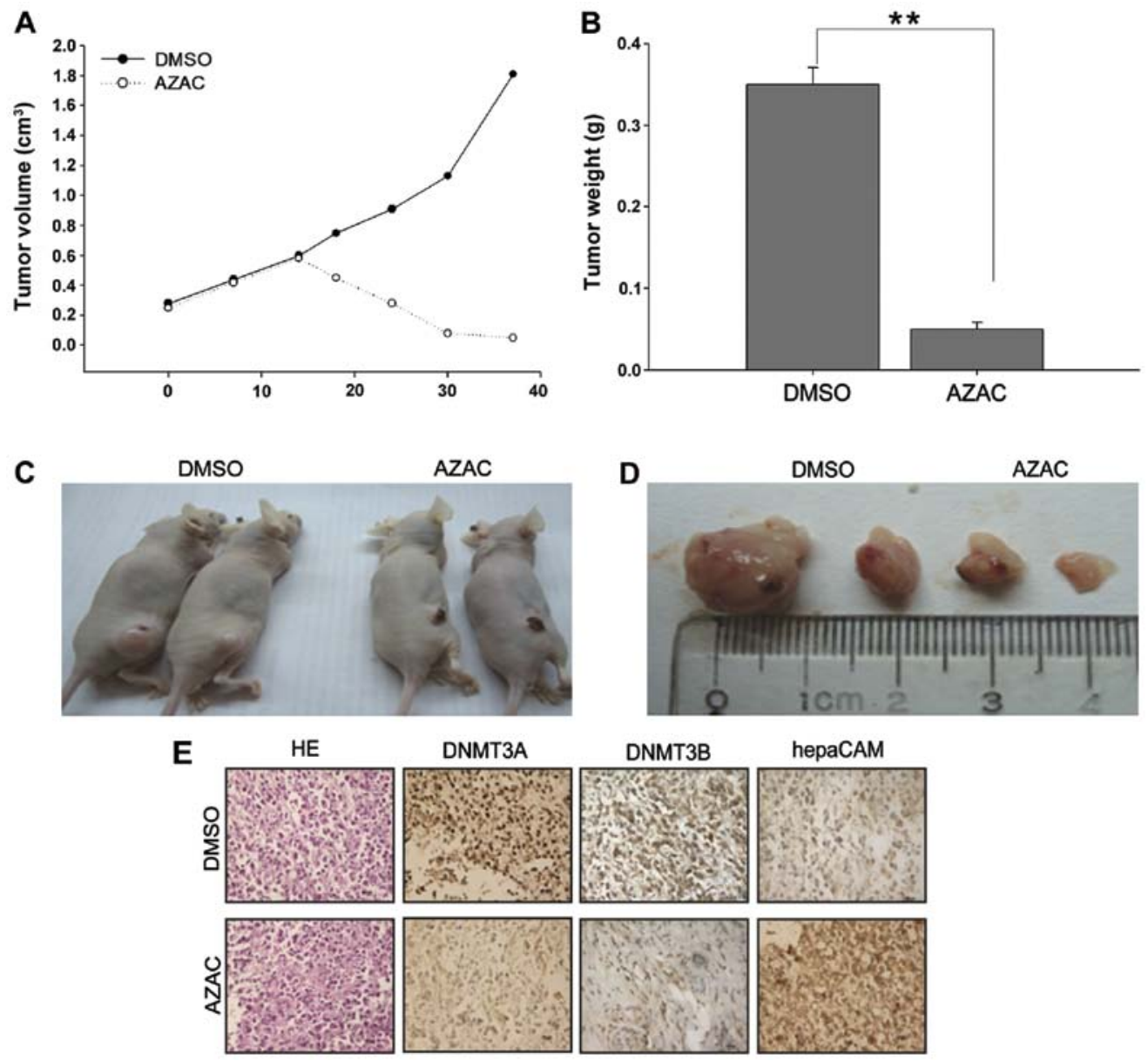

Figure 4. Effects of AZAC on the tumorigenicity of bladder cancer in vivo. (A) Growth curve of engrafted tumors in nude mice injected with EJ cells. (B) Tumor growth curve of the AZAC group in nude mice compared with the control tumors; ${ }^{* *} \mathrm{P}<0.01$. (C and D) Tumors derived from DMSO- and AZACtreated EJ cells in nude mice. (E) Representative images of hematoxylin and eosin (HE) staining and immunohistochemical (IHC) analysis of DNMT3A, DNMT3B and hepaCAM in tumor tissues of the nude mice. Magnification, $\mathrm{x} 100$.

DNMT3A/3B, at not only the mRNA but also the protein level, in the bladder cancer cell lines. Previous studies also showed similar findings in prostate cancer cells under mahanine treatment (37). These studies indicate that the precise mechanism of low hepaCAM expression in bladder cancer may be abnormal hypermethylation of hepaCAM induced by high levels of DNMT3A/3B.

In the clinic, AZAC has been used as a drug for hematopoietic disorders and as an anticancer treatment strategy for solid tumors. Fenaux et al showed that AZAC significantly extended the overall survival of patients with myelodysplastic syndrome (MDS, a bone marrow stem cell disorder). Likewise, AZAC was found to suppress the cell growth of three different neuroendocrine tumors in vitro (38). In the present study, CCK-8 and colony-formation assays revealed that AZAC inhibited the proliferation of bladder cancer cells. For the first time, we also provided evidence for the accumulation of G0/G1 cell cycle markers following AZAC treatment, suggesting that AZAC inhibited cell proliferation by inducing G0/G1 cell growth arrest. These data were in concordance with a previously published report that adenovirus-hepaCAM treatments induce G0/G1 growth arrest in bladder cancer (19). Thus, our data suggest that AZAC may represent an effective therapeutic intervention for bladder cancer.
To address the regulation mechanism and apparently central role of AZAC in vitro, we performed in vivo experiments to validate these results. First, in vivo tests revealed that AZAC inhibited tumor growth, which was in line with a previous study (39). Next, immunohistochemistry showed low protein levels of DNMT3A/3B and high protein expression of hepaCAM in the AZAC-treated tumors, which were in accordance with our in vitro tests. However, the sites of methylation of hepaCAM in bladder cancer cells need to be further explored.

In summary, our study showed that DNMT3A/3B expression was increased while hepaCAM protein expression was decreased in bladder cancer tissues, and there was a negative linear correlation between them. HepaCAM was silenced by its promoter hypermethylation and was re-activated by methylation inhibitor AZAC via downregulation of the expression of DNMT3A/3B. Furthermore, AZAC inhibits the growth of bladder cancer in vitro and in vivo, providing a new insight into the therapeutic strategy of bladder cancer.

\section{Acknowledgements}

This study was supported by the National Natural Science Foundation of China (grant no. 81072086). 


\section{References}

1. Favre-Kontula L, Rolland A, Bernasconi L, Karmirantzou M, Power C, Antonsson B and Boschert U: GlialCAM, an immunoglobulin-like cell adhesion molecule is expressed in glial cells of the central nervous system. Glia 56: 633-645, 2008.

2. Chung Moh M, Hoon Lee L and Shen S: Cloning and characterization of hepaCAM, a novel Ig-like cell adhesion molecule suppressed in human hepatocellular carcinoma. J Hepatol 42: 833-841, 2005.

3. Moh MC, Zhang C, Luo C, Lee LH and Shen S: Structural and functional analyses of a novel Ig-like cell adhesion molecule hepaCAM, in the human breast carcinoma MCF7 cells. J Biol Chem 280: 27366-27374, 2005.

4. Moh MC, Zhang T, Lee LH and Shen S: Expression of hepaCAM is downregulated in cancers and induces senescence-like growth arrest via a p53/p21-dependent pathway in human breast cancer cells. Carcinogenesis 29: 2298-2305, 2008.

5. Lee LH, Moh MC, Zhang T and Shen S: The immunoglobulin-like cell adhesion molecule hepaCAM induces differentiation of human glioblastoma U373-MG cells. J Cell Biochem 107: $1129-1138,2009$.

6. Xun C, Luo C, Wu X, Zhang Q, Yan L and Shen S: Expression of hepaCAM and its effect on proliferation of tumor cells in renal cell carcinoma. Urology 75: 828-834, 2010.

7. Zhang QL, Luo CL, Wu XH, Wang CY, Xu X, Zhang YY, Liu Q and Shen SL: HepaCAM induces G1 phase arrest and promotes c-Myc degradation in human renal cell carcinoma. J Cell Biochem 112: 2910-2919, 2011.

8. He Y, Wu X, Luo C, Wang L and Lin J: Functional significance of the hepaCAM gene in bladder cancer. BMC Cancer 10: 83, 2010.

9. Zhu X, Shan L, Wang F, Wang J, Wang F, Shen G, Liu X, Wang B, Yuan Y, Ying J, et al: Hypermethylation of BRCA1 gene: Implication for prognostic biomarker and therapeutic target in sporadic primary triple-negative breast cancer. Breast Cancer Res Treat 150: 479-486, 2015.

10. Ahmed IA, Pusch CM, Hamed T, Rashad H, Idris A, El-Fadle AA and Blin N: Epigenetic alterations by methylation of RASSF1A and DAPK1 promoter sequences in mammary carcinoma detected in extracellular tumor DNA. Cancer Genet Cytogenet 199: 96-100, 2010.

11. Liang G, Chan MF, Tomigahara Y, Tsai YC, Gonzales FA, Li E, Laird PW and Jones PA: Cooperativity between DNA methyltransferases in the maintenance methylation of repetitive elements. Mol Cell Biol 22: 480-491, 2002.

12. Ramos MP, Wijetunga NA, McLellan AS, Suzuki M and Greally JM: DNA demethylation by 5-aza-2'-deoxycytidine is imprinted, targeted to euchromatin, and has limited transcriptional consequences. Epigenetics Chromatin 8: 11, 2015.

13. Amara K, Ziadi S, Hachana M, Soltani N, Korbi S and Trimeche M: DNA methyltransferase DNMT3b protein overexpression as a prognostic factor in patients with diffuse large B-cell lymphomas. Cancer Sci 101: 1722-1730, 2010.

14. Chen MF, Chen WC, Chang YJ, Wu CF and Wu CT: Role of DNA methyltransferase 1 in hormone-resistant prostate cancer. J Mol Med Berl 88: 953-962, 2010.

15. Devanand P, Kim SI, Choi YW, Sheen SS, Yim H, Ryu MS, Kim SJ, Kim WJ and Lim IK: Inhibition of bladder cancer invasion by Sp1-mediated BTG2 expression via inhibition of DNA methyltransferase 1. FEBS J 281: 5581-5601, 2014.

16. Brueckner B and Lyko F: DNA methyltransferase inhibitors: Old and new drugs for an epigenetic cancer therapy. Trends Pharmacol Sci 25: 551-554, 2004.

17. Wijermans P, Lübbert M, Verhoef G, Bosly A, Ravoet C, Andre M and Ferrant A: Low-dose 5-aza-2'-deoxycytidine, a DNA hypomethylating agent, for the treatment of high-risk myelodysplastic syndrome: A multicenter phase II study in elderly patients. J Clin Oncol 18: 956-962, 2000.

18. Issa JP, Garcia-Manero G, Giles FJ, Mannari R, Thomas D, Faderl S, Bayar E, Lyons J, Rosenfeld CS, Cortes J, et al: Phase 1 study of low-dose prolonged exposure schedules of the hypomethylating agent 5-aza-2'-deoxycytidine (decitabine) in hematopoietic malignancies. Blood 103: 1635-1640, 2004.

19. Xu B, He Y, Wu X, Luo C, Liu A and Zhang J: Exploration of the correlations between interferon- $\gamma$ in patient serum and HEPACAM in bladder transitional cell carcinoma, and the interferon $-\gamma$ mechanism inhibiting BIU-87 proliferation. J Urol 188: 1346-1353, 2012.
20. Wang Q, Luo C, Wu X, Du H, Song X and Fan Y: hepaCAM and p-mTOR closely correlate in bladder transitional cell carcinoma and hepaCAM expression inhibits proliferation via an AMPK/mTOR dependent pathway in human bladder cancer cells. J Urol 190: 1912-1918, 2013.

21. Alexander VM, Roy M, Steffens KA, Kunnimalaiyaan M and Chen H: Azacytidine induces cell cycle arrest and suppression of neuroendocrine markers in carcinoids. Int J Clin Exp Med 3: 95-102, 2010

22. Pasin E, Josephson DY, Mitra AP, Cote RJ and Stein JP: Superficial bladder cancer: An update on etiology, molecular development, classification, and natural history. Rev Urol 10: 31-43, 2008.

23. Zeegers MP, Kellen E, Buntinx F and van den Brandt PA: The association between smoking, beverage consumption, diet and bladder cancer: A systematic literature review. World J Urol 21: 392-401, 2004.

24. Scrima M, De Marco C, De Vita F, Fabiani F, Franco R, Pirozzi G, Rocco G, Malanga D and Viglietto G: The nonreceptor-type tyrosine phosphatase PTPN13 is a tumor suppressor gene in non-small cell lung cancer. Am J Pathol 180: 1202-1214, 2012.

25. Yoon JI, Kim SI, Tommasi S and Besaratinia A: Organ specificity of the bladder carcinogen 4-aminobiphenyl in inducing DNA damage and mutation in mice. Cancer Prev Res (Phila) 5: 299-308, 2012.

26. Kandimalla R, van Tilborg AA and Zwarthoff EC: DNA methylation-based biomarkers in bladder cancer. Nat Rev Urol 10: 327-335, 2013.

27. Bilgrami SM, Qureshi SA, Pervez S and Abbas F: Promoter hypermethylation of tumor suppressor genes correlates with tumor grade and invasiveness in patients with urothelial bladder cancer. Springerplus 3: 178, 2014. doi: 10.1186/2193-1801-3-178.

28. Li G, Liu Y, Yin H,Zhang X, Mo X, Tang J and Chen W: E-cadherin gene promoter hypermethylation may contribute to the risk of bladder cancer among Asian populations. Gene 534: 48-53, 2014.

29. Piaton E, Casalegno JS, Advenier AS, Decaussin-Petrucci M, Mege-Lechevallier F, Ruffion A and Mekki Y: p16(INK4a) overexpression is not linked to oncogenic human papillomaviruses in patients with high-grade urothelial cancer cells. Cancer Cytopathol 122: 760-769, 2014.

30. Pan C, Wu X, Luo C, Yang S, Pu J, Wang C and Shen S: Exon 2 methylation inhibits hepaCAM expression in transitional cell carcinoma of the bladder. Urol Int 85: 347-354, 2010.

31. Jones PA and Baylin SB: The fundamental role of epigenetic events in cancer. Nat Rev Genet 3: 415-428, 2002.

32. Stutterheim J, Ichou FA, den Ouden E, Versteeg R, Caron HN, Tytgat GA and van der Schoot CE: Methylated RASSF1a is the first specific DNA marker for minimal residual disease testing in neuroblastoma. Clin Cancer Res 18: 808-814, 2012.

33. Tian X, Chen D, Zhang R, Zhou J, Peng X, Yang X, Zhang X and Zheng Z: Quantitative survey of multiple CpGs from 5 genes identifies $\mathrm{CpG}$ methylation panel discriminating between high- and low-grade cervical intraepithelial neoplasia. Clin Epigenetics 7: 4, 2015.

34. Jing H, Dai F, Zhao C, Yang J, Li L, Kota P, Mao L, Xiang K, Zheng $\mathrm{C}$ and Yang $\mathrm{J}$ : Association of genetic variants in and promoter hypermethylation of $\mathrm{CDH} 1$ with gastric cancer: $\mathrm{A}$ meta-analysis. Medicine (Baltimore) 93: e107, 2014.

35. Ding WJ, Fang JY, Chen XY and Peng YS: The expression and clinical significance of DNA methyltransferase proteins in human gastric cancer. Dig Dis Sci 53: 2083-2089, 2008.

36. He S, Wang F, Yang L, Guo C, Wan R, Ke A, Xu L, Hu G, Xu X, Shen J, et al: Expression of DNMT1 and DNMT3a are regulated by GLI1 in human pancreatic cancer. PLoS One 6: e27684, 2011.

37. Agarwal S, Amin KS, Jagadeesh S, Baishay G, Rao PG, Barua NC, Bhattacharya S and Banerjee PP: Mahanine restores RASSF1A expression by down-regulating DNMT1 and DNMT3B in prostate cancer cells. Mol Cancer 12: 99-110, 2013.

38. Fenaux P, Mufti GJ, Hellstrom-Lindberg E, Santini V, Finelli C, Giagounidis A, Schoch R, Gattermann N, Sanz G, List A, et al; International Vidaza High-Risk MDS Survival Study Group: Efficacy of azacitidine compared with that of conventional care regimens in the treatment of higher-risk myelodysplastic syndromes: A randomised, open-label, phase III study. Lancet Oncol 10: 223-232, 2009

39. Borodovsky A, Salmasi V, Turcan S, Fabius AW, Baia GS, Eberhart CG, Weingart JD, Gallia GL, Baylin SB, Chan TA, et al: 5-Azacytidine reduces methylation, promotes differentiation and induces tumor regression in a patient-derived IDH1 mutant glioma xenograft. Oncotarget 4: 1737-1747, 2013. 